\title{
Distributional Results for Dependent Type-II Hybrid Censored Order Statistics
}

\author{
Abbas Pak \\ Department of Computer Sciences, Faculty of Mathematical Sciences \\ Shahrekord University, P. O. Box 115, Shahrekord, Iran \\ abbas.pak1982@gmail.com \\ Hamid Dashti \\ Department of Statistics, Islamic Azad University, Tehran, Iran \\ hamidd361@gmai.com
}

\begin{abstract}
The scheme of type-II hybrid censoring is of great value in life-testing experiments. In the literature, type-II hybrid censored order statistics are assumed to arise from independent random variables. However, in real lifetime systems, it is quite common for the components to be dependent. In this paper, we study the properties of type-II hybrid censored order statistics in the case when the units are statistically dependent. Density, distribution and joint density functions of dependent type-II hybrid censored order statistics are derived under this set-up. For certain special cases, more explicit expressions are presented. Illustrative examples are also provided.
\end{abstract}

Keywords: Type-II hybrid censoring, Copula function, Order statistics, Completely monotone generator function.

\section{Introduction}

For manufacturers, assessing the reliability of an existing product is a fundamental task for improving the product's reliability or quality. However, due to cost and time consideration, it is difficult to observe all of the lifetime data of products within a reasonable period of time. Data obtained from such experiments are called censored sample. There are many types of censoring schemes used in lifetime analysis. The two most common censoring schemes are termed as type-I and type-II censoring schemes. In the conventional type-I censoring scheme, the experiment continues up to a pre-specified time $T$. On the other hand, the conventional type-II censoring scheme requires the experiment to continue until a pre-specified number of failures occur. A hybrid censoring scheme is a mixture of type-I and type-II censoring schemes, and it can be described as follows. Suppose $n$ identical units are put on a test. The test is terminated when a prechosen number $l$ out of $n$ items are failed, or when a pre-determined time $T$ on the test has been reached. Epstein (1954) introduced this type-I hybrid censoring scheme, and considered lifetime experiments assuming that the lifetime of each unit follows an exponential distribution.

Like the conventional type-I censoring scheme, the disadvantage of the type-I hybrid censoring scheme is that all the inferential results are obtained under the condition that the number of observed failures is at least one, and in addition there may be very few failures occurring up to the pre-fixed time $T$. Because of that, Childs et al. (2003) proposed a new hybrid censoring scheme known as the type-II hybrid censoring scheme, and it can be described as follows. Put $n$ identical items on test, and then stop the 
experiment at the random time $T^{*}=\max \left\{X_{l: n}, T\right\}$, where $X_{l: n}$ denotes the time of $l$-th failure. Thus, the type-II hybrid censoring scheme ensures that at least $l$ failures take place. The work on type-II hybrid censoring has become quite popular in life-testing and reliability studies. Childs et al. (2003) discussed exact likelihood inference based on typeII hybrid censored samples from the exponential distribution. Estimating the parameters of Weibull distribution under type-II hybrid censoring scheme is considered by Banerjee and Kundu (2008). Panahi and Asadi (2011) studied analysis of the type-II hybrid censored Burr type XII distribution under Linex loss function. Kohansal et al. (2015) considered the estimation of parameters of Weighted exponential distribution based on type-II hybrid censored data.

However, all aforementioned results have been developed under the key assumption that the units under test are independently distributed. In real lifetime systems, it is quite common for the components to be dependent. The main aim of this paper is to study type-II hybrid censored order statistics arising from dependent random variables. Copula theory is one of the best methods used for modeling the dependency in conventional works. Copulas are multivariate joint distributions of random variables with uniform marginal distributions. They have become increasingly important in statistical models in which the dependence structures cannot be simply described in terms of the classical Pearson correlation coefficient. In recent years, there has been a revival of copula in applications where the matter of dependency between random variables is of importance (see Bekrizadeh et al. (2012)).

In this paper, we consider type-II hybrid censored order statistics arising from dependent units that are jointly distributed by using some copula functions. In Section 2, we obtain the density and distribution functions of dependent type-II hybrid censored order statistics. The marginal density and distribution functions of the r-th order statistic as well as the joint density function of the $r$-th and s-th order statistics arising from dependent random variables are also derived in this section. In Section 3, an illustrative example is provided in which we consider order statistics arise from $n$ dependent variables distributed according to the generalized Gumbel Hougaard family of Archimedean copulas. Finally, conclusions are made in Section 4. Let us first review the main definitions of copula theory and some of the formula used in this paper.

The study of copula functions gives a fully developed mathematical theory for multivariate distribution analysis. A copula is a function that links univariate distribution functions to generate a multivariate distribution function and thus represents the dependency structure of random variables. In other words, copulas enable us to extract the dependence structure from joint distribution function of a set of random variables and, at the same time, to isolate the dependence structure from the univariate marginal behavior (Li and Sun (2009)). Copula functions have been extensively studied and a comprehensive discussion of their mathematical properties has been presented (see Joe (1997) and Nelsen (2006)).

The foundation theorem for copula was introduced by Sklar (1959) which states that for a given joint multivariate probability distribution function (pdf) and the relevant marginal pdfs, there exists a copula function that relates them. Let $\underline{X}=\left(X_{1}, \ldots, X_{n}\right)$ be a vector of 
$n$ continuous random variables with joint cumulative distribution function (cdf) denoted by $F_{\underline{X}}$ and univariate marginals $F_{X_{i}}, i=1, \ldots, n$. According to Sklar's theorem, $F_{\underline{X}}$ can be written as a function of the univariate marginals $F_{X_{i}}$ and the copula $C$ describing the dependence structure as follows:

$$
F_{\underline{X}}\left(X_{1}, \ldots, X_{n}\right)=C\left(F_{X_{1}}\left(x_{1}\right), \ldots, F_{X_{n}}\left(x_{n}\right)\right)
$$

By using (1), the joint probability density function of $\underline{X}$ can be obtained as

$$
f_{\underline{X}}\left(X_{1}, \ldots, X_{n}\right)=f_{X_{1}}\left(x_{1}\right) \ldots f_{X_{n}}\left(x_{n}\right) c\left(F_{X_{1}}\left(x_{1}\right), \ldots, F_{X_{n}}\left(x_{n}\right)\right)
$$

where $c$ is the corresponding pdf of the copula $C$ defined by

$$
c\left(u_{1}, \ldots, u_{n}\right)=\frac{\partial C\left(u_{1}, \ldots, u_{n}\right)}{\partial u_{1} \ldots \partial u_{n}} .
$$

One of the most important parametric family of copulas is the Archimedean copula. Archimedean copulas are popular because they are constructed easily and allow modeling the dependence in arbitrarily high dimensions with only one parameter, governing the strength of dependence. If a copula $C \varphi$ has the form

$$
C \varphi\left(u_{1}, \ldots, u_{n}\right)=\varphi\left(\sum_{i=1}^{n} \varphi^{-1}\left(u_{i}\right)\right),
$$

where $\varphi: \mathfrak{R}_{+} \rightarrow[0,1]$ is a $n$-monotone function such that $\varphi(0)=1$ and $\lim _{x \rightarrow \infty} \varphi(x)=0$, then it is called an Archimedean copula (Khoolenjani and Alamatsaz (2015)). $\varphi$ is said to be the generator function of this Archimedean copula. Let $G(u)=\exp \left\{-\varphi^{-1}(u)\right\}$ and $M \varphi$ be the distribution function with Laplace Transform $\varphi$. Then, we can obtain an equivalent representation of relation (3) as

$$
C \varphi\left(u_{1}, \ldots, u_{n}\right)=\int_{0}^{\infty} \prod_{i=1}^{n} G^{v}\left(u_{i}\right) d M \varphi(v)
$$

In the next section, we will use this representation of Archimedean copula to obtain the properties of type-II hybrid order statistics.

\section{Main results}

Suppose that $n$ identical units are placed on a life test with the corresponding lifetimes $X_{1}, \ldots, X_{n}$. It is assumed that these variables are dependent and identically distributed with a common absolutely continuous cumulative distribution function $F$ and probability density function $f$. In this section, we develop distributional results for type-II hybrid censored order statistics from $X_{1}, \ldots, X_{n}$. To be specific, we assume that the $n$ underling variables are jointly distributed according to an Archimedean copula with joint distribution function

$$
\varphi\left(\sum_{i=1}^{n} \varphi^{-1}\left(u_{i}\right)\right)=\int_{0}^{\infty} \prod_{i=1}^{n} G^{v}\left(F\left(x_{i}\right)\right) d M \varphi(v)
$$


Then, the joint density function of $\underline{X}=\left(X_{1}, \ldots, X_{n}\right)$ equals

$$
f_{\underline{X}}\left(x_{1}, \ldots, x_{n}\right)=\int_{0}^{\infty} \prod_{i=1}^{n} v g\left(F\left(x_{i}\right)\right) G^{v-1}\left(F\left(x_{i}\right)\right) f\left(x_{i}\right) d M \varphi(v)
$$

where

$$
g\left(F\left(x_{i}\right)\right)=-\frac{\exp \left(-\varphi^{-1}\left(F\left(x_{i}\right)\right)\right.}{\varphi^{\prime}\left(\varphi^{-1}\left(F\left(x_{i}\right)\right)\right.} .
$$

For known $m$ and $T$ and under the type-II hybrid censoring scheme, we can observe the following two types of observations:

Case I: $\left\{x_{1: n}, \ldots, x_{l: n}\right\}$ if $x_{l: n}>T$.

Case II: $\left\{x_{1: n}<\ldots<x_{l: n}<x_{l+1: n}<\ldots<x_{m: n}<T<x_{m+1: n}\right\}$ if $l \leq m$ and $x_{m: n}<T<x_{m+1: n}$.

Note that, in case II, we do not observe $x_{m+1: n}$, but $x_{m: n}<T<x_{m+1: n}$ means that the $m$-th failure took place before $T$, and no failure took place between $x_{m: n}$ and $T$. A schematic representation of the Type-II hybrid censoring scheme is presented in Fig. 1. In the following theorem, we derive the joint density function of the type-II hybrid censored order statistics.
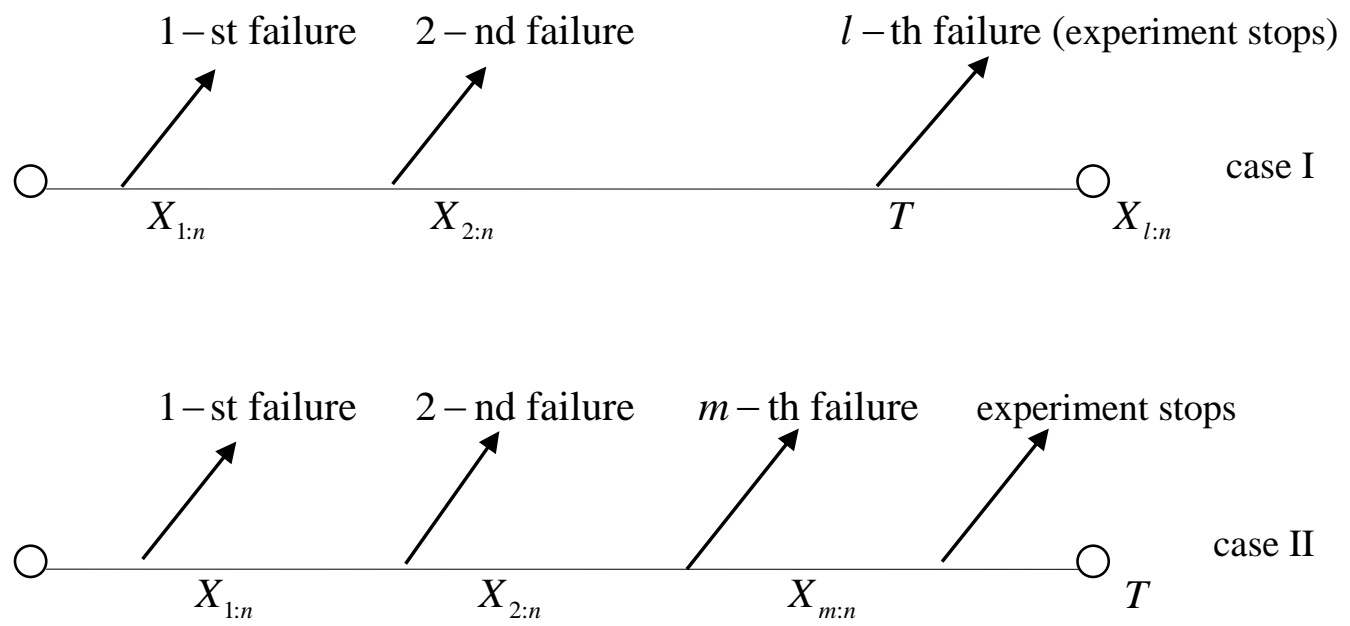

Figure 1. Schematic illustration of the type-II hybrid censoring scheme

Theorem 1: Suppose that $n$ dependent random variables $X_{1}, \ldots, X_{n}$ are jointly distributed according to an Archimedean copula with joint distribution function given in (4). Then, the joint density of order statistics from type-II hybrid censoring scheme is given by

$$
f_{\underline{X^{D}}}\left(x_{1}, \ldots, x_{D}\right)=\int_{0}^{\infty}\left(G^{v}(\bar{F}(u))\right)^{n-D} \prod_{i=1}^{D} v g\left(\bar{F}\left(x_{i}\right)\right) G^{v-1}\left(\bar{F}\left(x_{i}\right)\right) f\left(x_{i}\right) d M \varphi(v)
$$

where $D$ denotes the number of failures; and $u=x_{l: n}$ if $D=l$, and $u=T$ if $D>l$. 
Proof: The joint cumulative distribution function of the type-II hybrid censored order statistics for the two cases is obtained as

Case I:

$$
P\left(\underline{X^{l}} \leq\left(x_{1: n}, \ldots, x_{l: n}\right)\right)=\sum_{S_{n}} P\left(X_{i_{1}} \leq x_{1: n}, \ldots, X_{i_{l}} \leq x_{l: n}, X_{i_{l+1}}>x_{l: n}, \ldots, X_{i_{n}}>x_{l: n}\right),
$$

Case II:

$$
P\left(\underline{X^{m}} \leq\left(x_{1: n}, \ldots, x_{m: n}\right)\right)=\sum_{S_{n}} P\left(X_{i_{1}} \leq x_{1: n}, \ldots, X_{i_{m}} \leq x_{m: n}, X_{i_{m+1}}>x_{m: n}, \ldots, X_{i_{n}}>x_{m: n}\right),
$$

where the summation $S_{n}$ extends over all permutations $\left(i_{1}, \ldots, i_{n}\right)$ of $1, \ldots, n$. Combining the two cases, the above cdfs can be written as

$$
\begin{gathered}
P\left(\underline{X^{D}} \leq\left(x_{1: n}, \ldots, x_{D: n}\right)\right)=\sum_{S_{n}} P\left(X_{i_{1}} \leq x_{1: n}, \ldots, X_{i_{D}} \leq x_{D: n}, X_{i_{D+1}}>u, \ldots, X_{i_{n}}>u\right) \\
=\int_{-\infty}^{x_{1: n}} \ldots \int_{-\infty}^{x_{D: n}} P\left(X_{i_{D+1}}>u, \ldots, X_{i_{n}}>u \mid X_{i_{1}}=x_{1}, \ldots, X_{i_{D}}=x_{D}\right) \\
\times f_{X_{i_{1}}, \ldots, X_{i_{D}}}\left(x_{1}, \ldots, x_{D}\right) d x_{D} \ldots d x_{1} .
\end{gathered}
$$

By using relation (5), (8) can be expressed as

$$
P\left(\underline{X^{D}} \leq\left(x_{1: n}, \ldots, x_{D: n}\right)\right)=\int_{0}^{\infty} \int_{-\infty}^{x_{1}} \ldots \int_{-\infty}^{x_{D}}\left(G^{v}(\bar{F}(u))\right)^{n-D} \prod_{i=1}^{D} v g\left(\bar{F}\left(x_{i}\right)\right) G^{v-1}\left(\bar{F}\left(x_{i}\right)\right) f\left(x_{i}\right) d x_{D} \ldots d x_{1} d M \varphi(v) .
$$

Differentiation of the last term with respect to $x_{1}, \ldots, x_{D}$ yields the joint density function in (7) and the proof is completed.

It is interesting to note that, by using definition of the $i-$ th derivative of the generator function $\varphi$, an alternative representation of the joint density function of the type-II hybrid censored order statistics can be obtained as follows.

Corollary 2: Let $\varphi^{(D)}$ denotes the $D$-th derivative of $\varphi$ and $h_{i}(x)=\frac{f\left(x_{i}\right)}{\varphi^{\prime}\left(\varphi^{-1}\left(\bar{F}\left(x_{i}\right)\right)\right.}$. Then, the joint density function of order statistics from type-II hybrid censoring scheme can be expressed as

$$
f_{\underline{X^{D}}}\left(x_{1}, \ldots, x_{D}\right)=\prod_{i=1}^{D} h_{i}(x) \varphi^{(D)}\left\{(n-D) \varphi^{-1}\left(\bar{F}(u)+\sum_{i=1}^{D} \varphi^{-1}\left(\bar{F}\left(x_{i}\right)\right)\right\} .\right.
$$

Proof: Since $G($.$) forms a proper distribution function with density g($.$) , we can obtain$ the survival function $\bar{G}(., v)$ as

$$
\bar{G}\left(x_{i}, v\right)=G^{v}\left(\bar{F}\left(x_{i}\right)\right)=\exp \left(-v \varphi^{-1}\left(\bar{F}\left(x_{i}\right)\right)\right.
$$


Now let

$$
\begin{aligned}
g\left(x_{i}, v\right) & =v g\left(\bar{F}\left(x_{i}\right)\right) G^{v-1}\left(\bar{F}\left(x_{i}\right)\right) f\left(x_{i}\right) \\
& =-v \frac{f\left(x_{i}\right)}{\varphi^{\prime}\left(\varphi^{-1}\left(\bar{F}\left(x_{i}\right)\right)\right.} \bar{G}\left(x_{i}, v\right) .
\end{aligned}
$$

Then, we obtain from (7) that

$$
\begin{aligned}
f_{\underline{X^{D}}}\left(x_{1}, \ldots, x_{D}\right) & =\int_{0}^{\infty}\left(G^{v}(\bar{F}(u))\right)^{n-D} \prod_{i=1}^{D} v g\left(\bar{F}\left(x_{i}\right)\right) G^{v-1}\left(\bar{F}\left(x_{i}\right)\right) f\left(x_{i}\right) d M \varphi(v) \\
& =\int_{0}^{\infty}\left(G^{v}(\bar{F}(u))\right)^{n-D} \prod_{i=1}^{D} g\left(x_{i}, v\right) d M \varphi(v) \\
& =\int_{0}^{\infty}\left(G^{v}(\bar{F}(u))\right)^{n-D}(-v)^{D} \prod_{i=1}^{D} h_{i}(x) \bar{G}\left(x_{i}, v\right) d M \varphi(v) \\
& =\prod_{i=1}^{D} h_{i}(x) \int_{0}^{\infty}(-v)^{D} \exp \left(-v\left[(n-D) \varphi^{-1}(\bar{F}(u))+\sum_{i=1}^{D} \varphi^{-1}\left(\bar{F}\left(x_{i}\right)\right)\right]\right) d M \varphi(v) \\
& =\prod_{i=1}^{D} h_{i}(x) \varphi^{(D)}\left\{(n-D) \varphi^{-1}(\bar{F}(u))+\sum_{i=1}^{D} \varphi^{-1}\left(\bar{F}\left(x_{i}\right)\right)\right\}
\end{aligned}
$$

as required.

Remark3: Consider the type-II hybrid censored order statistics arising from identical and independent distributions. In this case, we have $\varphi(x)=\exp (-x)$ and $M \varphi(x)=1$ for $x=1$ and equals zero elsewhere. Then, by using (7), we arrive at the representation

$$
f_{\underline{X^{D}}}\left(x_{1}, \ldots, x_{D}\right)=\prod_{i=1}^{D} f\left(x_{i}\right)(\bar{F}(u))^{n-D} .
$$

Now, by using (7), we can obtain the marginal density and distribution functions of the $r$-th order statistic as well as the joint density and distribution functions of the $r$-th and $s$-th order statistics arising from $n$ dependent random variables that are jointly distributed according to an Archimedean copula. The one- and two-dimensional marginals have the density and distribution functions as given in the following two theorems.

Theorem 4: The probability density and distribution functions of the $r$-th order statistics arising from $n$ dependent random variables with joint survival function $\varphi\left(\sum_{i=1}^{n} \varphi^{-1}\left(\bar{F}\left(x_{i}\right)\right)\right)$, where $\varphi$ is a completely monotone function is expressed as

$$
f_{x_{r, n}}(x)=\sum_{i=1}^{r} \frac{f\left(x_{i}\right)}{\varphi^{\prime}\left(\varphi^{-1}\left(\bar{F}\left(x_{i}\right)\right)\right.} \varphi^{\prime}\left\{(n-v+1) \varphi^{-1}(\bar{F}(u))+\sum_{i=1}^{D} \varphi^{-1}\left(\bar{F}\left(x_{i}\right)\right)\right\}
$$


and

$$
F_{x_{r: n}}(x)=1-\sum_{i=1}^{r} \varphi\left\{(n-v+1) \varphi^{-1}(\bar{F}(u))+\sum_{i=1}^{D} \varphi^{-1}\left(\bar{F}\left(x_{i}\right)\right)\right\}
$$

Proof: The representation of the density function $f_{\underline{X^{r}}}\left(x_{1}, \ldots, x_{r}\right)$ given in Theorem 1 can be expressed as

$$
f_{\underline{X^{D}}}\left(x_{1}, \ldots, x_{D}\right)=\int_{0}^{\infty} f_{\underline{X_{v}}}\left(x_{1}, \ldots, x_{D}\right) d M \varphi(v)
$$

where $f_{\underline{X_{v}}{ }^{D}}\left(x_{1}, \ldots, x_{D}\right)$ is defined as the integrand in (8). Combining the mixing representation of the joint density function in (14) with the expression for the marginal density function in Kamps and Cramer (2001), we get

$$
\begin{aligned}
f_{x_{r: n}}(x) & =\int_{0}^{\infty} f_{x_{r n: n}}(x) d M \varphi(v)=\int_{0}^{\infty}\left(G^{v}(\bar{F}(u))\right)^{n-D} \sum_{i=1}^{D} g\left(x_{i}, v\right) \bar{G}^{v-1}\left(x_{i}, v\right) d M \varphi(v) \\
& =\sum_{i=1}^{D} \frac{f\left(x_{i}\right)}{\varphi^{\prime}\left(\varphi^{-1}\left(\bar{F}\left(x_{i}\right)\right)\right.} \int_{0}^{\infty}-v \bar{G}\left(x_{i}, v\right)\left(G^{v}(\bar{F}(u))\right)^{n-D} \bar{G}^{v-1}\left(x_{i}, v\right) d M \varphi(v) \\
& =\sum_{i=1}^{D} \frac{f\left(x_{i}\right)}{\varphi^{\prime}\left(\varphi^{-1}\left(\bar{F}\left(x_{i}\right)\right)\right.} \int_{0}^{\infty}-v \exp \left((n-v+1) \varphi^{-1}(\bar{F}(u))+\sum_{i=1}^{D} \varphi^{-1}\left(\bar{F}\left(x_{i}\right)\right) d M \varphi(v)\right. \\
& =\sum_{i=1}^{r} \frac{f\left(x_{i}\right)}{\varphi^{\prime}\left(\varphi^{-1}\left(\bar{F}\left(x_{i}\right)\right)\right.} \varphi^{\prime}\left\{(n-v+1) \varphi^{-1}(\bar{F}(u))+\sum_{i=1}^{D} \varphi^{-1}\left(\bar{F}\left(x_{i}\right)\right\} .\right.
\end{aligned}
$$

The corresponding distribution function in (13) can be obtained analogously.

The bivariate density function of $\left(X_{s: n}, X_{r: n}\right), 1 \leq s<r$, can be derived in a similar manner.

Theorem 4: Suppose that $n$ dependent random variables are jointly distributed according to an Archimedean copula with joint survival function $\varphi\left(\sum_{i=1}^{n} \varphi^{-1}\left(\bar{F}\left(x_{i}\right)\right)\right)$. Then, the bivariate probability density function of the $r$-th and $s$-th order statistics is expressed as

$$
\begin{aligned}
f_{x_{s: n}, x_{r \cdot n}}\left(x_{s}, x_{r}\right)=\sum_{j=s+1}^{r} \sum_{i=1}^{s} & \frac{f\left(x_{s}\right)}{\varphi^{\prime}\left(\varphi^{-1}\left(\bar{F}\left(x_{s}\right)\right)\right.} \frac{f\left(x_{r}\right)}{\varphi^{\prime}\left(\varphi^{-1}\left(\bar{F}\left(x_{r}\right)\right)\right.} \\
& \times \varphi^{(2)}\left\{(n-v+1) \varphi^{-1}\left(\bar{F}\left(x_{r}\right)\right)+v\left[\varphi^{-1}\left(\bar{F}\left(x_{i}\right)\right)-\varphi^{-1}\left(\bar{F}\left(x_{j}\right)\right)\right]\right\} .
\end{aligned}
$$


Proof: By (14), we can obtain the bivariate density function of the $r$-th and $s$-th order statistics as

$$
\begin{aligned}
f_{x_{s, n}, x_{r n}}\left(x_{s}, x_{r}\right) & =\int_{0}^{\infty} f_{x_{s n n ;},}, x_{r n: v}\left(x_{s}, x_{r}\right) d M \varphi(v) \\
& =\int_{0}^{\infty} G^{v(n-D)}\left(\bar{F}\left(x_{r}\right)\right) \sum_{j=s+1}^{r} \sum_{i=1}^{s} g\left(x_{i}, v\right) g\left(x_{j}, v\right) \bar{G}^{v-1}\left(x_{i}, v\right) d M \varphi(v) \\
& =\sum_{j=s+1}^{r} \sum_{i=1}^{s} \frac{f\left(x_{s}\right)}{\varphi^{\prime}\left(\varphi^{-1}\left(\bar{F}\left(x_{s}\right)\right)\right.} \frac{f\left(x_{r}\right)}{\varphi^{\prime}\left(\varphi^{-1}\left(\bar{F}\left(x_{r}\right)\right)\right.} \\
& =\sum_{j=s+1}^{r} \sum_{i=1}^{s} \frac{f\left(x_{s}\right)}{\varphi^{\prime}\left(\varphi^{-1}\left(\bar{F}\left(x_{s}\right)\right)\right.} \frac{f\left(x_{r}\right)}{\varphi^{\prime}\left(\varphi^{-1}\left(\bar{F}\left(x_{r}\right)\right)\right.} \\
& \times \int_{0}^{\infty} v^{2} \exp \left((n-v+1) \varphi^{-1}\left(\bar{F}\left(x_{r}\right)\right)+v\left[\varphi^{-1}\left(\bar{F}\left(x_{i}\right)\right)-\varphi^{-1}\left(\bar{F}\left(x_{j}\right)\right)\right]\right) d M \varphi(v)
\end{aligned}
$$

The desired result can be obtained, by using the definition of the second derivative of the generator function $\varphi$, as

$$
\begin{aligned}
f_{x_{s: n}, x_{r \cdot n}}\left(x_{s}, x_{r}\right)=\sum_{j=s+1}^{r} \sum_{i=1}^{s} & \frac{f\left(x_{s}\right)}{\varphi^{\prime}\left(\varphi^{-1}\left(\bar{F}\left(x_{s}\right)\right)\right.} \frac{f\left(x_{r}\right)}{\varphi^{\prime}\left(\varphi^{-1}\left(\bar{F}\left(x_{r}\right)\right)\right.} \\
& \times \varphi^{(2)}\left\{(n-v+1) \varphi^{-1}\left(\bar{F}\left(x_{r}\right)\right)+\varphi^{-1}\left(\bar{F}\left(x_{i}\right)\right)-\varphi^{-1}\left(\bar{F}\left(x_{j}\right)\right)\right\} .
\end{aligned}
$$

Thus, the proof is completed.

\section{An Illustrative example}

In this section, we provide an example in which $n$ dependent exponential random variables are distributed according to Archimedean copula with a completely monotone generator function.

Considering the generator function of Archimedean copula as $\varphi(t)=\exp \left(-t^{1 / \eta}\right)$, explicit expression for the joint density function of type-II hybrid censored order statistics can be derived. In this case, $\varphi^{-1}(t)=(-\log (t))^{\eta}, \bar{F}\left(x_{i}\right)=\exp \left(-\theta x_{i}\right), \theta>0$, and

$$
\begin{aligned}
\varphi^{\prime}\left(\varphi^{-1}\left(\bar{F}\left(x_{i}\right)\right)\right) & =\frac{1}{\eta}\left(\varphi^{-1}\left(\bar{F}\left(x_{i}\right)\right)\right)^{\frac{1}{\eta}-1} \varphi\left(\varphi^{-1}\left(\bar{F}\left(x_{i}\right)\right)\right) \\
& =\frac{1}{\eta}\left(\varphi^{-1}\left(\exp \left(-\theta x_{i}\right)\right)\right)^{\frac{1}{\eta}-1} \exp \left(-\theta x_{i}\right) \\
& =\frac{1}{\eta}\left(\left(\theta x_{i}\right)^{\eta}\right)^{\frac{1}{\eta}-1} \exp \left(-\theta x_{i}\right) \\
& =\frac{\left(\theta x_{i}\right)^{1-\eta}}{\eta} \exp \left(-\theta x_{i}\right) .
\end{aligned}
$$


Therefore, we have

$$
\begin{aligned}
G\left(\bar{F}\left(x_{i}\right)\right)= & G\left(\exp \left(-\theta x_{i}\right)\right) \\
& =\exp \left(-\varphi^{-1}\left(\exp \left(-\theta x_{i}\right)\right)\right) \\
& =\exp \left(-\left(-\log \left(\exp \left(-\theta x_{i}\right)\right)\right)^{\eta}\right) \\
& =\exp \left(-\left(\theta x_{i}\right)^{\eta}\right)
\end{aligned}
$$

and

$$
\begin{aligned}
g\left(\bar{F}\left(x_{i}\right)\right)= & g\left(\exp \left(-\theta x_{i}\right)\right) \\
= & -\frac{\exp \left(-\varphi^{-1}\left(\exp \left(-\theta x_{i}\right)\right)\right)}{\varphi^{\prime}\left(\varphi^{-1}\left(\exp \left(-\theta x_{i}\right)\right)\right)} \\
= & \frac{\exp \left(-\left(\theta x_{i}\right)^{\eta}\right)}{\frac{\left(\theta x_{i}\right)^{1-\eta}}{\eta} \exp \left(-\theta x_{i}\right)} \\
= & \eta\left(\theta x_{i}\right)^{\eta-1} \exp \left(\theta x_{i}\right) \exp \left(-\left(\theta x_{i}\right)^{\eta}\right) .
\end{aligned}
$$

Now, by using (7), the joint density function of the random vector $\left(X_{1: n}, \ldots, X_{D: n}\right)$ equals

$$
\begin{gathered}
f_{\underline{X^{D}}}\left(x_{1}, \ldots, x_{D}\right)=\int_{0}^{\infty}\left(G^{v}(\bar{F}(u))\right)^{n-D} \prod_{i=1}^{D} v g\left(\bar{F}\left(x_{i}\right)\right) G^{v-1}\left(\bar{F}\left(x_{i}\right)\right) f\left(x_{i}\right) d M \varphi(v) \\
=\int_{0}^{\infty}\left(\exp \left(-v(\theta u)^{\eta}\right)\right)^{n-D} \prod_{i=1}^{D} v \eta\left(1-\exp \left(-\theta x_{i}\right)\right)\left(\theta x_{i}\right)^{\eta-1} \exp \left(\theta x_{i}\right) \\
\quad \times \exp \left(-\left(\theta x_{i}\right)^{\eta}\right) \exp \left(-(v-1)\left(\theta x_{i}\right)^{\eta}\right) \exp \left(-\theta x_{i}\right) d M \varphi(v) \\
=\int_{0}^{\infty} \exp \left(-v(n-D)(\theta u)^{\eta}\right) v^{D} \eta^{D} \prod_{i=1}^{D}\left(1-\exp \left(-\theta x_{i}\right)\right)\left(\theta x_{i}\right)^{\eta-1} \\
\quad \times \exp \left(-v\left(\theta x_{i}\right)^{\eta}\right) d M \varphi(v) \\
\quad \eta^{D} \theta^{(\eta-1) D} \prod_{i=1}^{D}\left(1-\exp \left(-\theta x_{i}\right)\right) \quad \\
\quad \times \int_{0}^{\infty} v^{D} \exp \left(-v\left[(n-D)(\theta u)^{\eta}-\theta^{\eta} \sum_{i=1}^{D} x_{i}^{\eta}\right]\right) d M \varphi(v)
\end{gathered}
$$

Since $(-1)^{D} \varphi^{(D)}(t)=\int_{0}^{\infty} v^{D} \exp (-v t) d M \varphi(v)$, the joint density function of type-II hybrid censored order statistics $\left(X_{1: n}, \ldots, X_{D: n}\right)$ can be expressed as

$$
f_{\underline{X^{D}}}\left(x_{1}, \ldots, x_{D}\right)=\eta^{D} \theta^{(\eta-1) D} \prod_{i=1}^{D}\left(1-\exp \left(-\theta x_{i}\right)\right)(-1)^{D} \varphi^{(D)}\left(-v\left[(n-D)(\theta u)^{\eta}-\theta^{\eta} \sum_{i=1}^{D} x_{i}^{\eta}\right]\right) .
$$




\section{Conclusion}

In the context of life-testing, type-II hybrid censoring has been studied extensively. But, all the results have been developed under the key assumption that the units under test are independently distributed. In this paper, we consider type-II hybrid censored order statistics arising from dependent units that are jointly distributed according to an Archimedean copula. Some properties of dependent type-II hybrid censored order statistics are derived under this set-up. It is shown that the joint density function of the units can be expressed as a function of the $m$-th derivative of the completely monotone generator function $\varphi$. An explicit form for the marginal density and distribution function of the $r$-th order statistic as well as the joint density function of the $r$-th and $s$-th order statistics are also obtained. The previous results on independent order statistics is included as a special case.

\section{References}

1. Banerjee, A. and Kundu, D. (2008). Inference based on type-II hybrid censored data from a Weibull distribution. IEEE Transactions on reliability, 57(2), 369-378.

2. Bekrizadeh, H, Parham, G.A., and Zadkarmi, M.Z. (2012). Weighted CookJohnson Copula and their Characterizations: Application to Probably Modeling of the Hot Spring Eruptions. Journal of Modern Applied Statistical Methods, 11(2), 517-523.

3. Childs, A., Chandrasekhar, B., Balakrishnan, N. and Kundu, D. (2003). Exact likelihood inference based on type-I and type-II hybrid censored samples from the exponential distribution. Annals of the Institute of Statistical Mathematics, 55, 319-330.

4. Epstein, B. (1954). Truncated life tests in the exponential case. Annals of Mathematical Statistics, 25, 555-564.

5. Joe, H. (1997). Multivariate Models and Dependence Concepts. In Monographs on Statistics and Applied Probability, vol. 73. London: Chapman \& Hall.

6. Kamps, U. and Cramer, E. (2001). On distributions of generalized order statistics. Statistics, 35, 269-280.

7. Khoolenjani, N.B. and Alamatsaz, M.H. (2015). A De Bruijn's identity for dependent random variables based on copula theory. Probability in the Engineering and Informational Sciences, Doi: 10.1017/S0269964815000315.

8. Kohansal, A., Rezakhah, S. and Khorram, E. (2015). Parameter estimation of type-II hybrid censored Weighted exponential distribution. Communications in Statistics-Simulation and Computation. 44(5), 1273-1299.

9. Li, H. and Sun, Y. (2009). Tail dependence for heavy-tailed scale mixtures of multi-variate distributions. Journal of Applied Probability, 46, 925-937.

10. Nelsen, R.B. (2006). An Introduction to Copulas, 2nd edn. New York: Springer.

11. Panahi, H. and Asadi, S. (2011). Analysis of the type-II hybrid censored Burr type XII Distribution under Linex loss function. Applied Mathematical Sciences, 5(79), 3929-3942.

12. Sklar, A. (1959). Fonctions de repartition an dimensions et leurs marges. Publications de l'Institut de Statistique de l'Universite de Paris, 8, 229-231. 